\title{
MARKOV CELL STRUCTURES
}

\author{
BY F. T. FARRELL AND L. E. JONES ${ }^{1}$
}

Communicated by S. S. Chern, January 15, 1977

ABSTRACT. We show that the partition underlying a Markov partition for a dynamical system can be chosen to be a cell complex structure.

Let $M$ denote a Riemannian manifold of dimension $m, \Lambda$ a compact subset of $M$ lying in the interior of $M$, and $h: M \rightarrow M$ a diffeomorphism. Recall that $\Lambda$ is called a hyperbolic set for $h$ (see [6]) if

(a) $h: \Lambda \rightarrow \Lambda$ is a homeomorphism;

(b) $\left.T(M)\right|_{\Lambda}$ splits as a direct sum $\xi^{u} \oplus \xi^{s}$ of continuous subbundles;

(c) $D h\left(\xi^{u}\right)=\xi^{u}, D h\left(\xi^{s}\right)=\xi^{s}, D h$ is expansive on $\xi^{u}$ and contractive on $\xi^{s}$.

If $\Lambda=M$, then $h: M \rightarrow M$ is called an Anosov diffeomorphism. It is well known that the bundles $\xi^{u}, \xi^{s}$ integrate to give transversal foliations $W^{u}, W^{s}$ of M. (See [1].) Locally $W^{u}, W^{s}$ decompose $M$ into a cartesian product $\mathbf{R}^{k} \times \mathbf{R}^{l}$ where $k, l$ are the dimensions of the leaves in $W^{u}, W^{s}$, and $k+l=m$.

A cell structure for $W^{u}, W^{s}$ consists of a cell structure $C$ for $M$, such that each cell $\Delta \in C$ splits as a cartesian product of cells $\Delta=\Delta_{u} \times \Delta_{s}$ consistent with the local product structure given $M$ by $\left(W^{\mu}, W^{s}\right)$. We further require that if $\Delta \in C$ then each of $\partial \Delta_{u} \times \partial \Delta_{s}, \Delta_{u} \times \partial \Delta_{s}, \partial \Delta_{u} \times \Delta_{s}$ is a cellular subcomplex of $C$. Let $C^{i}, j$ denote the subset of $M$ equal the union of open cells

$$
\left\{\Delta \in C \mid \operatorname{dim}\left(\Delta_{u}\right) \leqslant i, \operatorname{dim}\left(\Delta_{s}\right) \geqslant j\right\}
$$

A Markov cell structure for an Anosov diffeomorphism $h: M \rightarrow M$ consists of a cell structure $C$ for $\left(W_{u}, W_{s}\right)$ satisfying $h^{n}\left(C^{i}, j\right) \subset C^{i}, j$ for all $i, j$ and some positive integer $n$.

THEOREM. There exist Markov cell structures for every Anosov diffeomorphism.

REMARKS. (1) A Markov cell structure for $h: M \rightarrow M$ is also a Markov partition for $h$, but not vice-versa. The partition sets of $M$ underlying a Markov partition of $h$, as defined in [5], will generally have nonfinitely generated homology groups.

(2) The theorem generalizes to give a Markov cell structure near any hyper-

AMS (MOS) subject classifications (1970). Primary 58F15.

${ }^{1}$ Both authors research were partially supported by grants from the National Science Foundation. 
bolic set $\Lambda$, which intersects $\Lambda$ in a Markov partition for $h: \Lambda \rightarrow \Lambda$ (as defined in [2]).

(3) In the theorem, $C$ car be cnosen so that the inclusion $C^{k}, l \subset C$ induces an epimorphism on integral homology groups through dimension $k$. By applying the constructions of [7] to $h: C^{k}, l \rightarrow C^{k}, l$, we obtain a commutative diagram

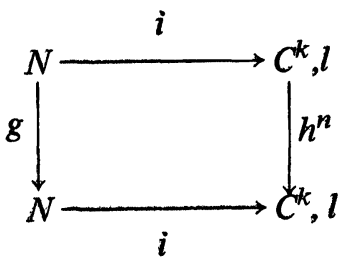

where $N$ is a compact differentiable branched manifold, $g$ is a differentaible expanding endomorphism, and $i$ is a homotopy equivalence. There is a cell structure on $N$ which makes $g^{s}$ a cellular map for some positive integer $s$.

(4) Using the setup in the previous remark, we can obtain by a purely geometric construction the dual homology classes associated to the transversal foliations $W^{u}, W^{s}$ which have been constructed in [3] by a combination of Markov partition and de Rham theory techniques. We can also prove that these dual classes are uniquely determined by their maximal expanding and minimal contracting properties. This uniqueness had already been proven in [4].

\section{BIBLIOGRAPHY}

1. D. V. Anosov, Geodesic flows on closed Riemann manifolds with negative curvature, Trudy Mat. Inst. Steklov 90 (1967), 209 pp. MR 36 \#157.

2. R. Bowen, Markov partitions for Axiom A diffeomorphisms, Amer. J. Math. 92 (1970), 725-747. MR 43 \#2740.

3. D. Ruelle and D. Sullivan, Currents, flows, and diffeomorphisms, Topology 14 (1975), 319-327.

4. M. Shub and R. Williams, Topology 14 (1975), 329-338.

5. I. G. Sinai, Markov partitions and C-diffeomorphisms, Funkcional Anal. i Priložen. 2 (1968), no. 1, 64-89. MR 38 \#1361.

6. S. Smale, Differentiable dynamical systems, Bull. Amer. Math. Soc. 73 (1967), 747-817. MR 37 \#3598.

7. R. Williams, Expanding attractors, Inst. Hautes Études Sci. Publ. Math. No. 43 (1974), 169-203. MR 50 \#1289.

DEPARTMENT OF MATHEMATICS, PENN STATE, UNIVERSITY PARK, PENNSYLVANIA 16802

DEPARTMENT OF MATHEMATICS, STATE UNIVERSITY OF NEW YORK, CENTER AT STONY BROOK, STONY BROOK, NEW YORK 11794 